\title{
Fractional flow reserve
}

\author{
Om Prakash Yadava ${ }^{1}$ (D) Filip Casselman ${ }^{2}$
}

Received: 22 June 2018 / Accepted: 13 July 2018 / Published online: 17 August 2018

(C) Indian Association of Cardiovascular-Thoracic Surgeons 2018

\begin{abstract}
Physiologically driven coronary revascularization has been shown to be superior in terms of better outcomes and optimum resource utilization for percutaneous interventions. However, its applicability to surgical myocardial revascularization lacks evidence base. GRAFITTI Trial aims at bridging this gap and Dr. F Casselman discusses its rationale and design.
\end{abstract}

Keywords Fractional flow reserve $\cdot$ GRAFITTI Trial $\cdot$ Revascularization

Dr. Casselman discusses (Video 1) the concept of fractional flow reserve (FFR) and the importance of looking at the physiological impact of the anatomical coronary artery stenosis. He elaborates the principles and rationale behind measurement and the critical value of 0.75 to 0.80 for FFR significance [1]. He goes on to discuss non-invasively measured computed tomography (CT)based FFR.

To a provocative question, is FFR an industry-driven investigation and is there any hard core data available for its use, Dr. Casselman feels that the jury is still divided but in the FAME Trial, though there was no mortality benefit, the clinical outcomes, apart from mortality, were superior with FFRbased percutaneous coronary intervention (PCI) [2]. In addition, the FAME II Trial was stopped prematurely because of overwhelming evidence in favor of FFR-based revascularization over optimal medical therapy. He also points that there is potential inter-observer variability in determination of FFR [3].

Electronic supplementary material The online version of this article (https://doi.org/10.1007/s12055-018-0709-0) contains supplementary material, which is available to authorized users.

Om Prakash Yadava

op_yadava@yahoo.com

1 National Heart Institute, New Delhi, India

2 OLV Clinic, Aalst, Belgium
Dr. Casselman discusses the rationale and the design of the forthcoming GRAFITTI Trial which compares angiographically driven coronary artery bypass graft(CABG) versus FFR-driven CABG. The inclusion criteria mandate a significant left anterior descending (LAD) lesion (FFR positive at 0.75 ) and an angiographically moderate lesion in either Circumflex or right coronary artery (RCA). The patients are then randomized into either a FFR or an angiography-guided surgical myocardial revascularization with regard to the circumflex $(\mathrm{Cx})$ and RCA territories. The primary end point is the graft patency at 1 year, as detected by coronary $\mathrm{CT}$ angiography. If there is a problem with the graft, then a routine cine angiography is performed [4].

Dr. Yadava discusses the confounding variables of OnPump versus Off-Pump and Minimally Invasive versus Sternotomy options that are permitted in the trial and their possible impact on final results.

\section{References}

1. Tonino PA, De Bruyne B, Pijls NH, et al. Fractional flow reserve versus angiography for guiding percutaneous coronary intervention. N Engl J Med. 2009;360:213-24.

2. van Nunen LX, Zimmermann FM, Tonino PA, et al. Fractional flow reserve versus angiography for guidance of PCI in patients with multivessel coronary artery disease (FAME): 5-year follow-up of a randomized controlled trial. Lancet. 2015;386:1853-60.

3. De Bruyne B, Fearon WF, Pijls NH, et al. Fractional flow reserveguided PCI for stable coronary artery disease. N Engl J Med. 2014;371:1208-17.

4. Toth GG, De Bruyne B, Kala P, et al. Study design of the graft patency after FFR-guided versus angiography-guided CABG trial (GRAFFITI). J Cardiovasc Trnasl Res. 2018. https://doi.org/10. 1007/s12265-018-9818-9. 